\title{
La Enseñanza de la Justicia Ambiental en el Marco de la Educación para el Desarrollo Sostenible en la Universidad
}

\author{
Teaching Environmental Justice in the Framework for \\ Education for Sustainable Development in College
}

\author{
María Martínez Lirola * \\ Universidad de Alicante, España \\ University of South Africa, República de Sudáfrica
}

\begin{abstract}
Uno de los retos actuales de la educación universitaria tiene que ver con la sostenibilidad y la justicia ambiental. Por esta razón se diseñaron distintas actividades relacionadas con la educación para la sostenibilidad con el fin de que el alumnado universitario pudiera reflexionar sobre la importancia de velar por el planeta, potenciar el pensamiento crítico y adquirir competencias sociales que sean de utilidad para el mercado laboral como la comunicación, la cooperación o la empatía. Dichas actividades se enmarcaron en una metodología cooperativa de modo que el alumnado tenía que trabajar en pequeños grupos para lograr un objetivo común. Al final del cuatrimestre se preparó una encuesta anónima para conocer la opinión del alumnado sobre determinados aspectos relacionados con la sostenibilidad y las distintas actividades llevadas a cabo. Los resultados de la encuesta muestran que el alumnado considera importante introducir temas relacionados con la justicia ambiental en la enseñanza universitaria. Además, señalan que han aprendido vocabulario y aspectos relacionados con la ecología y la sostenibilidad al realizar las actividades propuestas además de adquirir las competencias sociales señaladas.
\end{abstract}

Descriptores: Educación para el desarrollo sostenible; Enseñanza; Educación superior; Inglés; Justicia social.

\begin{abstract}
One of the current challenges of higher education has to do with sustainability and environmental justice. For this reason, different activities related to sustainable education were designed so that college students could reflect on the importance of watching over the planet, fostering critical thinking and acquiring social skills that would be useful for the labor market, such as communication, cooperation or empathy. These activities were framed in a cooperative methodology so that students had to work in small groups to achieve a common goal. At the end of the semester an anonymous survey was prepared in order to know students' opinion about certain aspects related to sustainability and the different activities carried out. The results of the survey show that students consider important to introduce issues related to environmental justice in university education. They also note that they have learned vocabulary and aspects related to ecology and sustainability in carrying out the proposed activities in addition to acquiring the mentioned social skills.
\end{abstract}

Keywords: Education for sustainable development; Teaching; Higher education; English; Social justice.

*Contacto: maria.lirola@ua.es

ISSN: 2254-3139

www.rinace.net/riejs/

revistas.uam.es/riejs
Recibido: $\quad 14$ de septiembre 2017

$1^{\text {a }}$ Evaluación: 13 de noviembre 2017

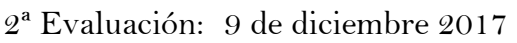

Aceptado: $\quad 2$ de enero 2018 


\section{Introducción}

Es bien sabido que todo aprendizaje tiene su origen en un entorno social y, por tanto, el entorno social mismo justifica los principios pedagógicos y sociales en los que se basa el aprendizaje en un contexto determinado. La educación del siglo XXI debe establecer lazos de unión entre educación y sociedad de modo que lo que ocurre en las aulas no está aislado de lo que ocurre en la sociedad (Martínez Lirola, 2017). Por tanto, se potencia que el alumnado pueda ver una relación directa entre lo que aprende y el contexto social en que se enmarca su aprendizaje. Gracias a la educación, las personas desarrollan sus capacidades y son capaces de comprender las distintas situaciones que les rodean de modo que avanzan como ciudadanía global que se adapta a los cambios que requiere la globalización (Boni y Calabuig, 2017; Boni, López-Fogues y Walker, 2016).

En todas las sociedades la educación ha de ser uno de los pilares fundamentales. En este sentido, el profesorado ha de apostar por una educación comprometida con la realidad social, que contribuya activamente a dar respuesta a las demandas sociales y al desarrollo de un mundo mejor. De este modo se potencia una educación holista (Espino de Lara, 2007) gracias a la cual el alumnado no adquiere solo conocimientos relacionados con el área de estudio sino también competencias y valores que le serán de gran utilidad tanto para su carrera profesional como para todos los aspectos de la vida.

La educación es, sin duda, una gran herramienta para contribuir a que las personas crezcan como ciudadanía crítica y formada en valores que fomenten la convivencia pacífica (Martínez Lirola, 2017). Concretamente la educación integral se centra tanto en la adquisición de conocimientos como en el componente afectivo con el fin de potenciar las distintas facetas de las personas, tal y como afirma Touriñán López (2008),

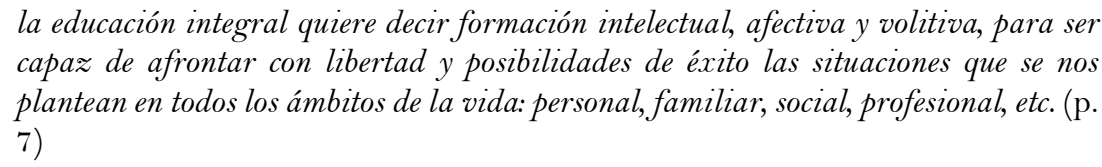

Optar por una educación integral implica que se potencia el pensamiento crítico y que las personas asumen un compromiso como ciudadanía activa y comprometida con la construcción de un mundo mejor. Una de las acciones concretas para el alumnado tiene que ver con velar por la justicia ambiental debido al deterioro que el planeta ha sufrido en los últimos años. Dentro de los distintos enfoques educativos que conceden importancia a la transformación social, la educación para el desarrollo sostenible (EDS en adelante) es la que se centra especialmente en el uso adecuado de los recursos de los que dispone el planeta y en el respeto al medio ambiente. Nos encontramos por tanto ante una propuesta educativa que potencia el cuidado y el respeto, aspectos esenciales para que se pueda avanzar en justicia social, que es sin duda uno de los retos fundamentales del siglo XXI.

Hemos de destacar la necesidad de una alfabetización crítica que lleve consigo la humanización y la transformación del mundo, es decir, se ha de formar a la ciudadanía de forma crítica para que se produzca una verdadera transformación social y se avance en la construcción de una auténtica democracia (Freire, 2001a, 2001b, 2015). También es fundamental establecer relaciones claras y directas entre el proceso de enseñanzaaprendizaje y el contexto social en que éste se enmarca con el objetivo de que la educación dé respuestas a las necesidades sociales. 
En este sentido, concurrimos con la siguiente afirmación de Barahona, Gratacós y Quintana (2013):

$$
\begin{aligned}
& \text { La perspectiva de una ciudadanía global plantea un desarrollo centrado en el ser } \\
& \text { humano integral, caracterizado, por encima de todas las cosas, por su capacidad } \\
& \text { crítica para desvelar la realidad y reconocer la diferencia entre lo que 'es' y lo que } \\
& \text { 'debería ser'. Consecuentemente, reconoce que ese 'debería ser' se ha de construir desde } \\
& \text { una doble perspectiva: primero, desde un diálogo participativo en el que nadie } \\
& \text { imponga su visión sobre las otraspersonas; y, segundo, desde una consciencia histórico- } \\
& \text { política de implicarse en la construcción de una sociedad más justa, más libre y más } \\
& \text { solidaria, que beneficie sobre todo a las mayorías excluidas en cada contexto y en el } \\
& \text { entorno global. Ese es el sentido de la transformación social. (p. 14) }
\end{aligned}
$$

Con el fin de potenciar el pensamiento crítico, este artículo está enmarcado en líneas internacionales en educación como las cuestiones socio científicas (CSC) que potencian trabajar con temas actuales y emergentes de nuestra sociedad como por ejemplo con el cambio climático, el calentamiento global, la comida, la calidad del aire, el consumo responsable o la biodiversidad (Duso y Bialvo, 2016; Solber, 2013).

Las CSC van unidas a los asuntos que generan conflicto y opiniones contrapuestas denominadas cuestiones socialmente vivas -questiones socialmente vives (QSV)-(Legardez y Simmoneaux, 2006), es decir, se trata de introducir en la enseñanza cuestiones que no están definidas ni por la ciencia ni por la sociedad. Esto lleva consigo que se potencie el debate y la controversia al tratar temas como la inmigración o la globalización. En consecuencia, introducir las QSV en el aula potencia la literacidad crítica del alumnado y la capacidad para reflexionar sobre los temas sociales seleccionados (Ortega Sánchez y Pagés Blanch, 2017).

Además de trabajar la capacidad crítica del alumnado, en todo momento del proceso de enseñanza-aprendizaje se potencia la relación entre la comunicación, lo que se aprende y la vida real (Lemke, 2012; Levine y Scollon, 2004; Tan, Kindelan y O'Halloran, 2012). De esta manera, el alumnado es consciente de que lo que aprende tiene una aplicación práctica pues los contenidos están relacionados con la realidad social, tal y como podremos ver en las actividades enmarcadas en el enfoque de la EDS que se presentarán en la sección 4 de este artículo. Por tanto, el aprendizaje se entiende como interactivo, dinámico y activo para el alumnado. Esto lleva consigo que además de conocimientos, el alumnado adquiere competencias sociales. La siguiente definición de competencia social ofrecida por Bisquerra y Pérez (2007) parece muy clara: "la capacidad para mantener buenas relaciones con otras personas. Esto implica dominar las habilidades sociales, capacidad para la comunicación efectiva, respeto, actitudes pro-sociales, asertividad" (p. 72).

Las preguntas de investigación que nos planteamos son las siguientes: ¿qué tipo de actividades pueden contribuir a desarrollar la justicia social en una asignatura de lengua inglesa en la educación superior? y ¿qué tipo de competencias desarrolla el alumnado al llevar a cabo las actividades seleccionadas?

Uno de los objetivos principales de este artículo es hacer una propuesta de actividades prácticas relacionadas con la justicia ambiental y la EDS que se han llevado a cabo en una asignatura de lengua inglesa en el grado de Estudios Ingleses. Otro objetivo es conocer la opinión del alumnado universitario que participó en las actividades diseñadas por lo que se diseñó una encuesta (ver Apéndice 1).

Además, en este artículo se observará la relación entre la enseñanza del inglés, sin duda una lengua global en la actualidad, y la introducción en el aula de temas globales 
relacionados con la sostenibilidad y la justicia ambiental. Trabajar con estos temas determina el tipo de vocabulario y los campos semánticos que se emplearán en el aula teniendo en cuenta las actividades diseñadas.

\section{Revisión de la literatura}

Existen distintos problemas ambientales que afectan tanto al planeta como a la vida de los seres humanos, entre los que destacan los siguientes: agotamiento de los recursos, pérdida de diversidad biológica y cultural, contaminación, degradación de los ecosistemas y conflictos. En este sentido, concurrimos con la siguiente afirmación de Cantú-Martínez (2014): "la degradación ambiental que actualmente se suscita se ha erguido como uno de los problemas más significativos, particularmente desde las postrimeras décadas del siglo XX” (p. 40).

La educación para el desarrollo sostenible fomenta que en el proceso de enseñanzaaprendizaje se traten temas relacionados con la importancia de la sostenibilidad, el cambio climático o la biodiversidad, entre otros. Se invita al alumnado de los distintos niveles educativos a que se comprometa con el respeto al medio ambiente de modo que se fomente el desarrollo de sociedades más sostenibles en las que las personas puedan tener mejor calidad de vida. Uno de los objetivos fundamentales de este enfoque educativo consiste en prestar atención al estado del planeta y en favorecer prácticas que fomenten la sostenibilidad y la ecología (Vilches y Gil Pérez, 2016).

Siguiendo a Riechmann (1995), el concepto de desarrollo sostenible aparece por primera vez en 1987 en el informe del Brundtland de la Comisión Mundial sobre el Medio Ambiente y Desarrollo titulado Nuestro futuro común. Este concepto no afecta sólo al medio ambiente, sino que en él también están presentes otras dimensiones como la económica, la educativa o la social (Aznar, 2010; Flórez-Yepes, 2015), por lo que se invita a que las personas participen activamente en distintos aspectos sociales para poder contribuir a la transformación de la sociedad y a la construcción de un mundo mejor. En definitiva, favorecer el desarrollo sostenible lleva consigo un estilo de vida en el que los valores juegan un papel importante (Bernardo y Tarí, 2013).

Trabajar por un planeta más sostenible implica que las personas potencian valores y actitudes con el fin de contribuir activamente a que el mundo sea más ecológico, sostenible, habitable y humano (UNESCO, 2014a, 2014b). En definitiva, se trata de apoyar un cambio de mentalidad para que las personas tomen conciencia de la grave situación en que se encuentra el planeta y de la importancia de asumir una responsabilidad global, siguiendo a Gómez Gómez y Ruiz Ávalos (2016),

los compromisos que, como sociedad tenemos, deben encaminarse al beneficio del conjunto en un futuro cercano, porque ahora se comprende mejor que no somos una comunidad aislada, pertenecemos a un entorno global donde lo que hagamos repercute en otros, la formación de los ciudadanos debe ser encaminada hacia la productividad y el desarrollo social. (p. 226)

La UNESCO (2015) diferencia los términos "desarrollo sostenible" y "sostenibilidad". Se refiere al primer término como el conjunto de medios y procesos para alcanzar la "meta a largo plazo" con que se refiere al segundo término.

La EDS dota a los estudiantes de la capacidad necesaria para tomar decisiones fundamentadas y realizar actividades responsables en pro de la integridad 
medioambiental, la viabilidad económica y la justicia social, para las generaciones actuales y las venideras, con el debido respeto a la diversidad cultural. (p. 12)

En los últimos años se han llevado a cabo numerosos estudios relacionados con la necesidad de introducir temas relacionados con la justicia ambiental y la sostenibilidad en los currícula (Aramburuzabala, Cerrillo y Tello, 2015; Lansu et al., 2013; Mayor Zaragoza, 2009; Murga-Menoyo, 2009; Vilches y Gil Pérez, 2015, 2016, entre otros).

Por un lado, hay estudios que se centran en América Latina (Flórez-Yepes, 2015; Macedo y Salgado, 2007); por otro lado, otras investigaciones se han llevado a cabo en distintas universidades europeas (Alfalla-Luque, Medina-López y Arenas-Márquez, 2011; Aznar et al., 2011; Lozano, 2010; Queiruga et al., 2015). Todos los estudios coinciden en la importancia de tratar en las aulas de los diferentes niveles educativos la importancia de velar por el planeta, de trabajar por el bien común y por la justicia social y ambiental para que la sociedad mejore y se pueda tener una calidad de vida digna en el planeta Tierra.

\section{Contexto, participantes y metodología}

Las actividades que se presentan en la sección que sigue se han puesto en práctica en el grado en Estudios Ingleses de la Universidad de Alicante, en concreto en la asignatura obligatoria Lengua Inglesa $\mathrm{V}$ que se imparte en tercer curso. Dicha asignatura tiene como objetivo fundamental que el alumnado desarrolle las distintas destrezas (escucha, habla, lectura, escritura e interacción) para alcanzar un nivel C1 dentro del Marco Común Europeo de Referencia de las Lenguas (Consejo de Europa, 2001). La asignatura se imparte durante cuatro horas semanales en el primer cuatrimestre. Una de ellas está dedicada a la presentación por grupos de una presentación oral sobre un tema de actualidad. Otra se dedica a profundizar en la forma y función de distintos tipos de textos. Semanalmente se trabajan temas de gramática de nivel C1 y finalmente, la última hora se dedica a que el alumnado desarrolle las destrezas orales al participar en un debate sobre un tema social.

En esta asignatura se destaca que la lengua y la sociedad están interrelacionadas, es decir, se potencia que el discurso que emplean los distintos miembros de una sociedad o las distintas instituciones es una práctica social. Se destaca que las personas hacen cosas a través del discurso pues cada vez que se habla o se escribe se hace con una función específica. El discurso también nos permite comunicar nuestra identidad y nuestra realidad social (Richardson, 2007).

El total de personas matriculadas en la asignatura durante el curso académico 2016-2017 fue de 102; el 87\% eran mujeres frente al 13\% de hombres. El alumnado tenía un nivel B2 (Consejo de Europa, 2001) tras haber estudiado inglés en los dos años anteriores de la carrera. La mayor parte del alumnado quería dedicarse a dar clase de inglés en el extranjero, en España como profesorado de secundaria o a trabajar en empresas internacionales.

Una propuesta educativa en favor de la justicia ambiental y la EDS ha de estar enmarcada en una metodología activa que facilite tanto potenciar el pensamiento crítico en el alumnado como el intercambio de opiniones. En este sentido, hemos optado por una metodología cooperativa que consistía en que el alumnado trabajara en pequeños grupos con el fin de alcanzar un objetivo común. De esta forma se puede profundizar en distintos temas sociales, como los relacionados con la justicia ambiental que se presentan en este 
artículo, a la vez que se facilita la reflexión y el diálogo. Así, se potencia la conciencia social a la vez que la comunicación debido a que las/os estudiantes tienen que interaccionar durante todo el proceso de enseñanza-aprendizaje con el fin de comprender mejor lo que ocurre en la sociedad y pensar posibles soluciones que pudieran contribuir a mejorar la sociedad en general y la justicia ambiental en particular.

Al principio del cuatrimestre, la profesora pidió al alumnado que se organizara en pequeños grupos de unas 4 o 5 personas para llevar a cabo distintas actividades cooperativas durante el cuatrimestre. Cada grupo tenía que contar con una persona que lo coordinara y que serviría de enlace entre el grupo y la profesora. En dichos grupos era fundamental que todos los miembros asumieran un compromiso activo para desarrollar las tareas que había que realizar durante el cuatrimestre. Así, cada miembro es consciente de que es responsable del éxito del resultado final.

La profesora diseñó distintas actividades enmarcadas en la educación para la ciudadanía global, prestando especial atención a la sostenibilidad, la igualdad de género y la interculturalidad. Por razones de espacio relacionadas con este artículo y por la temática del monográfico en que se enmarca, en la sección que sigue, vamos a presentar sólo actividades relacionadas con la justicia ambiental y presentaremos el resto de las actividades en otros artículos. Estas actividades contribuyen a que el alumnado sea consciente de los problemas ambientales y de la importancia de velar por la ecología y la sostenibilidad con el fin de que el planeta no se siga deteriorando.

La profesora diseñó una encuesta con el fin de conocer la opinión del alumnado universitario sobre determinados aspectos relacionados con la justicia social y la EDS. Dicha encuesta se analizó al final del cuatrimestre. Mientras el alumnado desarrollaba las distintas actividades, la profesora optó por la observación participante pues en todo momento estuvo en contacto con los grupos para resolver posibles dudas o dificultades. Anotó aspectos relacionados con la participación de los distintos miembros de los grupos, las dificultades observadas para llevar a cabo las tareas y la división cooperativa del trabajo.

La evaluación de las distintas actividades propuestas se llevó a cabo teniendo en cuenta los siguientes aspectos. En el caso concreto de las actividades orales era necesario que todo el alumnado participara y expresara su opinión en un inglés correcto. En el caso de las actividades escritas (el esquema, la carta al editor de un periódico y las normas para el buen funcionamiento de los grupos), los criterios de evaluación se centraron en el uso adecuado de la sintaxis y la gramática inglesa y en la creatividad y originalidad que el alumnado había mostrado al terminar las tareas.

\section{Resultados}

En esta sección se presentan los resultados obtenidos. Por un lado, se presentan como resultados cualitativos las actividades realizadas en clase para potenciar la educación para el desarrollo sostenible. Por otro lado, se presentan los datos obtenidos de la encuesta que contestó el alumnado, pues se trata de resultados cuantificables.

\subsection{Actividades que potencian la educación para el desarrollo sostenible}


Las actividades que proponemos en esta sección facilitan la reflexión sobre los problemas socio-ambientales que se observan en el planeta tanto a nivel local como global. Las actividades que siguen pretenden concienciar sobre la importancia del respeto al medio ambiente al potenciar el consumo responsable y la conservación de los recursos naturales, entre otros. En definitiva, se trata de que el alumnado se conciencie de que solo es posible conseguir una sociedad más justa a todos los niveles, incluido el ambiental, si se avanza en sostenibilidad.

El respeto al medio ambiente ocupa un lugar importante en la EDS y está entre los Objetivos del Desarrollo Sostenible (ODS) en los que las sociedades han de avanzar hasta el año 2030 (Naciones Unidas, 2015). Por esta razón, la profesora decidió comenzar esta actividad compartiendo los ODS para que el alumnado pudiera reflexionar sobre los mismos y decidir cuáles se relacionaban con las distintas actividades presentadas durante el cuatrimestre, de ahí que se presentara en clase la lista con los 17 objetivos $^{1}$ que ofrecemos a continuación:

- Erradicar la pobreza en todas sus formas en todo el mundo.

- Poner fin al hambre, conseguir la seguridad alimentaria y una mejor nutrición, y promover la agricultura sostenible.

- Garantizar una vida saludable y promover el bienestar para todos para todas las edades.

- Garantizar una educación de calidad inclusiva y equitativa, y promover las oportunidades de aprendizaje permanente para todos.

- Alcanzar la igualdad entre los géneros y empoderar a todas las mujeres y niñas.

- Garantizar la disponibilidad y la gestión sostenible del agua y el saneamiento para todos.

- Asegurar el acceso a energías asequibles, fiables, sostenibles y modernas para todos.

- Fomentar el crecimiento económico sostenido, inclusivo y sostenible, el empleo pleno y productivo, y el trabajo decente para todos.

- Desarrollar infraestructuras resilientes, promover la industrialización inclusiva y sostenible, y fomentar la innovación.

- Reducir las desigualdades entre países y dentro de ellos.

- Conseguir que las ciudades y los asentamientos humanos sean inclusivos, seguros, resilientes y sostenibles.

- Garantizar las pautas de consumo y de producción sostenibles.

- Tomar medidas urgentes para combatir el cambio climático y sus efectos (tomando nota de los acuerdos adoptados en el foro de la Convención Marco de las Naciones Unidas sobre el Cambio Climático).

- Conservar y utilizar de forma sostenible los océanos, mares y recursos marinos para lograr el desarrollo sostenible.

${ }^{1}$ Estos objetivos se pueden encontrar en el siguiente enlace: http://www.un.org/sustainabledevelopment/es/ 
- Proteger, restaurar y promover la utilización sostenible de los ecosistemas terrestres, gestionar de manera sostenible los bosques, combatir la desertificación y detener y revertir la degradación de la tierra, y frenar la pérdida de diversidad biológica.

- Promover sociedades pacíficas e inclusivas para el desarrollo sostenible, facilitar acceso a la justicia para todos y crear instituciones eficaces, responsables e inclusivas a todos los niveles.

- Fortalecer los medios de ejecución y reavivar la alianza mundial para el desarrollo sostenible.

Tras leer con detalle los distintos objetivos, se hace hincapié en que los objetivos 13 y 15 se desarrollan con la EDS y la justicia ambiental, temática de este artículo. Una vez hecho esto, la profesora pide al alumnado que en los distintos grupos formados al principio del cuatrimestre reflexione sobre los principales problemas ambientales a nivel global y a nivel local y se les invita a ofrecer posibles soluciones a los mismos con el fin de que se puedan ofrecer soluciones basadas en la ecología y el respeto al medio ambiente. A continuación, se solicita al alumnado que enumere cuántas organizaciones conoce relacionadas con la ecología y si participan activamente en alguna de ellas. El alumnado menciona Green Peace, WWF o Ecologistas en Acción y alrededor de un 20\% del alumnado señala que colabora asiduamente con ellas.

Seguidamente, la profesora comunica al alumnado que la siguiente actividad consiste en que por grupos preparen un esquema con algunas ideas y prácticas sobre cómo se puede mejorar el medio ambiente por medio de acciones concretas que se puedan llevar a cabo en nuestra cotidianidad. Cada grupo comparte las ideas de modo que toda la clase se enriquece con las propuestas, entre las que destacan:

- Ahorrar energía en casa apagando las luces que no se necesiten, usando la luz del día siempre que sea posible, moderando la temperatura de la calefacción o aire acondicionado, usando bombillas de bajo consumo, desenchufando los electrodomésticos que no se estén utilizando, etc.

- Utilizar el agua de forma razonable, por ejemplo, duchándose en vez de darse un baño, no dejar el grifo abierto mientras nos lavamos los dientes, evitar que haya grifos que goteen, no tirar innecesariamente de la cadena, etc.

- Ahorrar papel por lo que es necesario reciclar todo lo que se pueda, aprovechar el dorso de las hojas y escribir en todo el espacio en blanco. Una vez que el papel no nos haga falta es necesario reciclarlo en el contenedor azul.

- Consumir de forma responsable de modo que no se compren cosas que no hacen falta. En la medida de lo posible es bueno usar productos ecológicos, con poco embalaje o con un embalaje fácil de reciclar. Reutiliza las bolsas de plástico todas las veces que sea posible o utiliza bolsas de tela.

- Tener presentes las tres "r": reduce los deshechos que produces y utiliza las cosas todo el tiempo que puedas; reutiliza todo lo que sea posible, por ejemplo, los tarros de cristal sirven para guardar comida, las camisetas o sábanas viejas se pueden usar como trapos para limpiar; recicla separando los residuos orgánicos del vidrio, el papel o el cartón y el plástico. También es necesario reciclar los 
residuos tóxicos como las pilas o los medicamentos depositándolos en el contenedor correspondiente.

Como se puede ver, son muchas las ideas que ofrece el alumnado. Al tratarse de un campo semántico específico, al igual que en otras actividades, la profesora pide al alumnado que prepare un glosario con todos los términos relacionados con este tema. Si hay términos que no conocen, como pueden ser desarrollo sostenible, impacto medioambiental o consumo responsable entre otros, la profesora les remite a los diferentes diccionarios en papel y electrónicos que les recomendó al principio del cuatrimestre de modo que el alumnado sea autónomo en su aprendizaje y haga uso de los distintos recursos que tiene a su disposición para que el aprendizaje sea efectivo.

A continuación, la profesora explica que van a seguir trabajando en el desarrollo de la destreza escrita un poco más, de ahí que la siguiente actividad que las/os estudiantes tienen que realizar consista en escribir una carta al editor de un periódico pidiendo a los y las lectores/as que tomen conciencia sobre la necesidad de llevar a cabo acciones en el día a día con el fin de mejorar el planeta, ahorrar recursos, reutilizar todo lo que se pueda, etc. De esta forma se pide que las/os estudiantes escriban un texto con una función determinada y que se pueda emplear en un contexto real.

La profesora también comenta al alumnado que sería conveniente que redactaran unas normas que les parezcan necesarias para el buen funcionamiento de los grupos y de toda la clase; la profesora precisa que sería bueno que en esas normas estuvieran presentes cuestiones relacionadas con la ecología, tema sobre el que se está trabajando. De este modo, se pueden tener presentes aspectos relacionados con el uso razonable de los recursos en el proceso de enseñanza-aprendizaje como puede ser entregar los trabajos en papel reciclado impreso por las dos caras o no encender las luces en el aula siempre que se vea bien con la luz natural, entre otros. También se invita al alumnado a que vea las normas que redacta como responsabilidades y que trate de relacionarlas con lo que se les requerirá al incorporarse al mercado laboral.

\subsection{Resultados cuantificables}

Al final del cuatrimestre se preparó una encuesta anónima para que el alumnado que había participado durante el cuatrimestre en las actividades relacionadas con la justicia social pudiera expresar su opinión sobre los aspectos que se detallan a continuación.

Con respecto a la primera pregunta, el 72\% del alumnado encuestado considera que sí les parece adecuado introducir temas relacionados con la justicia ambiental y la sostenibilidad en la enseñanza universitaria con independencia de la carrera que se estudie por varias razones: porque les parece que es un tema que afecta a todas las personas, porque así se aprenden cosas nuevas, porque en el caso del grado en Estudios Ingleses se aprende vocabulario específico y porque les interesan los temas sociales. El $28 \%$ que ofreció una respuesta negativa la justifica porque considera que estos temas deben tratarse con detalle en los grados en con los que tienen relación como pueden ser el de Medio Ambiente o Ciencias de la Tierra. También señalan que cada carrera ha de centrarse en temas de su especialidad por lo que no les parece conveniente que se traten en el grado que estudian que se centra en los Estudios Ingleses.

Nos parece interesante constatar que, al plantear la segunda pregunta, el 93\% del alumnado señala que no conocía los ODS con anterioridad a que fueran mencionados en esta asignatura. Esto contrasta con los resultados obtenidos en la tercera pregunta donde 
el $84 \%$ de los/as estudiantes afirman que se consideran personas preocupadas por el medio ambiente. Las principales razones que ofrecen para justificar su respuesta positiva son las siguientes: porque reciclan, son voluntarios/as en asociaciones relacionadas con la ecología como Ecologistas en Acción, WWF o Green Peace o porque participan en acciones puntuales que tengan que ver con la mejora del medio ambiente.

Casi el mismo porcentaje, en concreto el $91 \%$ de las personas que responden la encuesta, afirma que sí ha aprendido vocabulario y aspectos relacionados con la ecología y la sostenibilidad al realizar las actividades propuestas. Esto confirma que trabajar con temas específicos en una asignatura de lengua inglesa favorece que el alumnado adquiera vocabulario específico y pueda emplearlo tanto al hablar como al escribir en inglés de modo que el vocabulario nuevo se integra con el desarrollo de distintas destrezas. Al analizar la última pregunta observamos que el $77 \%$ considera que ha adquirido por igual las tres competencias sociales que se han trabajado al realizar las actividades enmarcadas en la EDS. Un $2 \%$ ha señalado la empatía, un $8 \%$ ha marcado la cooperación y el $13 \%$ restante ha seleccionado sólo la comunicación.

\section{Discusión}

Proponer actividades sociales relacionadas con la JA en una asignatura universitaria de inglés permite relacionar las capacidades sociales de comunicación propias de una asignatura de lengua extranjera con el desarrollo del pensamiento crítico. En este estudio se han presentado actividades para que el alumnado mejore las distintas destrezas en inglés (escritura, escucha, habla, lectura e interacción) a la vez que trabaja la capacidad crítica, pues el alumnado tiene que reflexionar sobre los ODS y profundizar en las distintas problemáticas que hay detrás de los mismos. En definitiva, se proponía al alumnado ser crítico con el sistema pues los problemas sociales son tantos en la actualidad, incluidos los ambientales, que es necesario ofrecer espacios en las aulas universitarias para ser críticos con el modelo existente y reflexionar sobre otras alternativas.

Las actividades desarrolladas contribuyen a introducir algunas CSC en el aula de inglés como lengua extranjera. Esto lleva consigo que el alumnado ha de utilizar vocabulario relacionado con los temas actuales relacionados con la JA y la EDS seleccionados y ha de ser crítico y justificar sus reflexiones sobre dicho tema. Concurrimos con Solber (2013) y con Toledo y otros (2015) en que trabajar con las CSC puede contribuir a paliar el déficit de pensamiento crítico en nuestra sociedad además de favorecer en el alumnado la capacidad para argumentar y debatir.

Se ha considerado fundamental trabajar con problemáticas socioambientales en una clase universitaria de lengua inglesa con el fin de potenciar tanto la capacidad crítica del alumnado universitario como la capacidad para debatir sobre las QSV que son polémicas y que permiten que el alumnado exprese opiniones diversas. En este sentido, entendemos que la docencia de la lengua inglesa en la actualidad ha de ir más allá de la enseñanza de cuestiones gramaticales o léxicas al potenciar la formación del alumnado como ciudadanía crítica. Tal y como afirma Moreno-Fernández (2015) "es necesario una educación ambiental, que conlleva implícita e ineludiblemente la necesidad de educar ciudadanos participativos y comprometidos con su entorno y, por lo tanto, con su realidad" (p. 174).

Trabajar con el alumnado aspectos relacionados con la justicia ambiental va unido al desarrollo de una conciencia social y global que el alumnado podrá aplicar cuando se 
incorpore al mercado laboral. Es decir, se invita al alumnado a que amplíe su perspectiva y crezca en valores y en conciencia social de modo que avance como ciudadanía global. El tipo de actividades presentadas puede contribuir a desarrollar la justicia social en una asignatura de lengua inglesa en la educación superior debido a la temática que se trata. Son actividades cooperativas pues éstas facilitan el intercambio de opiniones y favorecen tanto la reflexión individual como la grupal además de potenciar el pensamiento crítico. En definitiva, se trata de que la clase fomente cambios sociales que contribuyan a una sociedad mejor, donde la justicia ambiental juega un papel importante (Mogensen et al., 2009).

Al igual que en otras investigaciones (Medir, Heras y Magin, 2016; de Paz Abril, 2013) vemos como una necesidad trabajar con un modelo educativo humanista y global, que ayude al alumnado a no solo adquirir conocimientos sino también a conceder importancia a la participación activa, crítica y democrática en la sociedad. Concurrimos con los diferentes estudios relacionados con la enseñanza de aspectos relacionados con la JA en el marco de la EDS en que se ha de tratar de un proceso continuo y permanente en el que el objetivo es la formación integral de la persona.

Tras lo expuesto en los párrafos anteriores, nos parece fundamental señalar que la educación integral contribuye a que las personas amplíen su perspectiva de la realidad y por tanto se fomenta que el alumnado tome conciencia de la importancia de avanzar como ciudadanía global, de modo que se asume una responsabilidad en los asuntos globales. En este sentido, al analizar la encuesta, nos parece significativo que la gran mayoría del alumnado encuestado está de acuerdo en que se trabaje sobre sostenibilidad y justicia ambiental en el aula pues gracias a ello las personas encuestadas consideran que aprenden vocabulario, profundizan en temas que les parecen de interés general y adquieren competencias sociales. El alumnado muestra un gran interés por estos temas y en general se consideran personas preocupadas por el medio ambiente como se puede ver claramente en sus respuestas. Los resultados de la encuesta se relacionan con otras investigaciones. Por ejemplo, concurrimos con Azcárate, Salvador y García (2012), al considerar que es necesario aumentar el nivel de inclusión de la sostenibilidad en los currícula universitarios. Esto lleva consigo que el alumnado amplía su conciencia y su capacidad crítica, a la vez que se favorece la adquisición de competencias (Lambrechts et al., 2013).

Las distintas investigaciones sobre la EDS señalan la necesidad de fomentar las competencias que conceden importancia al desarrollo sostenible con el fin de que el alumnado se conciencie de que velar por la justicia ambiental es un tema urgente. Con el fin de fomentar este tipo de competencias en la enseñanza superior proponemos actividades como las presentadas en la sección anterior.

Con respecto al tipo de competencias que el alumnado desarrolla al llevar a cabo las actividades seleccionadas destacan las competencias sociales como la empatía que ayuda al alumnado a reflexionar y tomar conciencia sobre la gravedad de los problemas ambientales que tiene el planeta. La cooperación es una competencia fundamental cuando se trabaja en pequeños grupos pues se ha de colaborar con el objetivo de realizar las actividades y lograr el objetivo común. La comunicación es otra competencia que se trabaja con las actividades presentadas pues el alumnado tiene que dialogar con sus iguales en todo momento del proceso de enseñanza-aprendizaje; también se potencia la comunicación escrita. 
Además de las competencias mencionadas, las actividades descritas potencian el desarrollo de las destrezas que se han de trabajar en lengua inglesa pues se ofrecen oportunidades para que el alumnado interactúe con sus iguales, de modo que se trabajan las destrezas relacionadas con el habla, la escucha y la interacción, además de poder leer y escribir textos en la lengua extranjera. Nos parece importante que la escritura esté unida a situaciones sociales reales de modo que al alumnado le resulte sencillo contextualizar lo que escribe al verle un claro propósito social.

También se concede importancia al desarrollo de la conciencia global que facilita la empatía con distintas problemáticas sociales, entre ellas la ambiental. En este sentido, la educación apuesta por una formación integral que concede protagonismo al alumnado en la construcción de un mundo mejor.

Se potencia el interés por la justicia social, incluida la ambiental, con el fin de que en el proceso de enseñanza-aprendizaje se trabaje con temas sociales que hagan al alumnado tomar conciencia de las injusticias que hay en el mundo y reflexionar sobre posibles soluciones. Por tanto, el papel que se concede al alumnado es activo para que se sienta libre para participar activamente en las actividades propuestas y para interpretar las realidades sociales que se tratan en el aula de manera crítica y creativa. Así, se fomentan las relaciones igualitarias en el aula pues el alumnado y el profesorado comparten la responsabilidad.

La metodología educativa que hemos propuesto y la temática de las actividades seleccionadas pueden contribuir a hacer frente a los problemas ambientales pues se considera fundamental abordar problemas inmediatos que afecten al medio ambiente en la enseñanza universitaria. Este tipo de tareas contribuyen a que el alumnado pueda reflexionar sobre las causas de los problemas ambientales del planeta y pensar en posibles soluciones que contribuyan a frenar el deterioro ambiental. En definitiva, se trata de potenciar la justicia ambiental al respetar la naturaleza y todas las especies que en ella habitan.

La justicia ambiental es un tema global que debería introducirse de manera transversal en la educación superior con el fin de que el alumnado tome conciencia de la importancia de la sostenibilidad y de la responsabilidad que cada ser humano tiene en velar por una vida digna en el planeta. En este sentido, introducir los oDs en el aula nos parece acertado pues ofrece la oportunidad de reflexionar sobre dichos objetivos e invita al alumnado a ofrecer propuestas que puedan contribuir a que se vaya avanzado en los distintos objetivos.

Consideramos que introducir temas relacionados con la justicia social y ambiental ayuda al alumnado a potenciar el pensamiento crítico y que, por tanto, estos temas se pueden introducir en todas las asignaturas de todos los niveles educativos. Sin duda, introducir temas sociales en el aula contribuye a que el alumnado amplíe su visión y le ayuda a reflexionar sobre la responsabilidad individual y colectiva que cada persona tiene en velar por una sociedad más justa y sostenible donde la vida sea el pilar fundamental.

\section{Referencias}

Alfalla-Luque, R., Medina-López, C. y Arenas-Márquez, F. J. (2011). Mejorando la formación en dirección de operaciones: La visión del estudiante y su respuesta ante diferentes metodologías docentes. Cuadernos de Economía y Dirección de la Empresa, 14, 40-52. https://doi.org/10.1016/j.cede.2011.01.002 
Aramburuzabala, P., Cerrillo, R. y Tello, I. (2015). Aprendizaje-servicio: Una propuesta metodológica para la introducción de la sostenibilidad curricular en la universidad. Profesorado, Revista de Currículum y Formación del Profesorado, 19(1), 78-95.

Azcárate, P., Salvador, A. N., y García, E. (2012). Aproximación al nivel de inclusión de la sostenibilidad en los curricula universitarios. Profesorado, Revista de Currículum y Formación del Profesorado, 16(2), 105-119.

Aznar, P. (2010). Educación para el desarrollo sostenible: Reflexiones teóricas y propuestas para la acción. EDETANIA, 37, 129-148.

Aznar, P., Martínez-Agut, M., Palacios, B., Piñero, A. y Ull, A. (2011). Introducing sustainability into university curricula: An indicator and baseline survey of the views of university teachers at the University of Valencia. Environmental Education Research, 17(2), 145-166. https://doi.org/10.1080/13504622.2010.502590

Barahona, R., Gratacós, J. y Quintana, G. (2013). Centros educativos transformadores. Ciudadanía global y transformación social. Barcelona: Oxfam Intermón.

Bernardo, M. y Tarí, J. (2013). Propuesta de código ético para la asignatura de gestión de la calidad: comparativa interuniversitaria. Revista de Educación en Contabilidad, Finanzas y Administración de Empresas (Educade), 4, 111-123.

Bisquerra, R. y Pérez, N. (2007). Las competencias emocionales. Educación XXI, 10, 61-82. https://doi.org/10.5944/educxx 1.1.10.297

Boni, A. y Calabuig, C. (2017). Education for global citizenship at universities. Journal of Studies in International Education, 21(1), 22-38. https://doi.org/10.1177/1028315315602926

Boni, A., López-Fogues, A. y Walker, M. (2016). Higher education and the post-2015 agenda: A contribution from the human development approach. Journal of Global Ethics, 12(1), 17-28. https://doi.org/10.1080/17449626.2016.1148757

Cantú-Martínez, P. C. (2014). Educación ambiental y la escuela como espacio educativo para la promoción de la sustentabilidad. Revista Electrónica Educare, 18(3), 39-52. https://doi.org/10.15359/ree.18-3.3

Consejo de Europa. (2001). Common European framework of reference for languages: Learning, teaching, assessment. Cambridge: Cambridge University Press.

Duso, L. y Bialvo, M. (2016). Discutiendo controversias socio científicas en la enseñanza de las ciencias por medio de una actividad lúdica. Revista Electrónica Universitaria de Formación del Profesorado, 19(2), 185-193.

Espino de Lara, R. (2007). Educación holista. Revista Iberoamericana de Educación, 44(1), 61-68.

Flórez-Yepes, G. Y. (2015). La educación ambiental y el desarrollo sostenible en el contexto colombiano. Revista Electrónica Educare, 19(3), 1-12. https://doi.org/10.15359/ree.19-3.5

Freire, P. (2001a). Pedagogía de la indignación. Madrid: Ediciones Morata.

Freire, P. (2001b). Pedagogia dos sonhos possíveis. Sao Paulo: Unesp.

Freire, P. (2015). Pedagogía liberadora. Madrid: Los libros de la Catarata.

Gómez Gómez, K. y Ruiz Ávalos, F.G. (2016). La dimensión del desarrollo sostenible en la creación de nueva oferta educativa para la Universidad Pedagógica Nacional. Uaricha, 13(30), 225249.

Lambrechts, W., Mulà, I., Ceulemans, K., Molderez, I. y Gaeremynck, V. (2013). The integration of competences for sustainable development in higher education: An analysis of bachelor 
programs in management. Journal of Cleaner Production, 48, 65-73. https://doi.org/10.1016/j.jclepro.2011.12.034

Lansu, A., Boon, J., Sloep, P. y Van Dam-Mieras, R. (2013). Changing professional demands in sustain-able regional development: A curriculum design process to meet transboundary

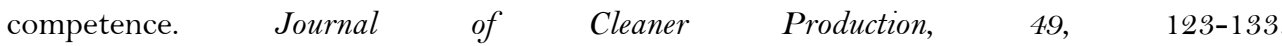
https://doi.org/10.1016/j.jclepro.2012.10.019

Legardez. A. y Simmoneaux, L. (Coords.). (2006). L'école á l'épreuve de la actualité. Enseigner les questions vives. París: ESF.

Lemke, J. L. (2012). Multimedia and discourse analysis. En J. P. Gee y M. Handford (Eds.), The Routledge handbook of discourse analysis (pp. 79-89). Londres: Routledge.

Levine, P. y Scollon, R. (Eds.) (2004). Discourse and technology. Multimodal discourse analysis. Washington, DC: Georgetown University Press.

Lozano, R. (2010). Diffusion of sustainable development in universities curricula: An empirical example from Cardiff University. Journal of Cleaner Production, 18, 637-644. https://doi.org/10.1016/j.jclepro.2009.07.005

Macedo, B. y Salgado, C. (2007). Educación ambiental y educación para el desarrollo sostenible en América Latina. Fórum de Sostenibilidad, 1, 29-37.

Martínez Lirola, M. (2017). La importancia del análisis crítico del discurso y la gramática visual para analizar textos. Propuesta de actividades prácticas enmarcadas en la educación para el desarrollo, la educación con perspectiva de género y la educación para la paz. Granada: Comares.

Mayor Zaragoza, F. (2009). La problemática de la sostenibilidad en un mundo globalizado. Revista de Educación, núm. extraordinario, 25-52.

Medir, R. M., Heras, R. y Magin, C. (2016). Una propuesta evaluativa para actividades de educación ambiental para la sostenibilidad. Educación XX1, 19(1), 331-355.

Mogensen, F., Mayer, M., Breiting, S. y Varga, A. (2009). Educación para el desarrollo sostenible. Barcelona: Graó.

Moreno-Fernández, O. (2015). Problemáticas socioambientales desde un enfoque de ciudadanía planetaria en las aulas. Revista de Humanidades, 24, 169-192. https://doi.org/10.5944/rdh.24.2015.15345

Murga-Menoyo, M. A. (2009). La carta de la tierra: Un referente de la década por la educación para el desarrollo sostenible. Revista de Educación, núm. extraordinario, 239-262.

Naciones Unidas. (2015) Objetivos de desarrollo sostenible. A/69/L.85. Recuperado de http://www.un.org/sustainabledevelopment/es/

de Paz Abril, D. (2013). Escuelas y educación para la ciudadanía global. Una mirada transformadora. Barcelona: Intermón Oxfam.

Ortega Sánchez, D. y Pagés Blanch, J. (2017). Literacidad crítica, invisibilidad social y género en la formación del profesorado de educación primaria. Revista de Investigación en Didáctica de las Ciencias Sociales, 1, 102-117.

Queiruga, D., González Benito, J, Amira, L. y Lannelongue, G. (2015). Educación para el desarrollo sostenible en asignaturas de dirección de operaciones. El caso del banco de alimentos de La Rioja. Working Papers on Operations Management, 6(1), 22-37.

Richardson, J. E. (2007). Analysing newspapers. An approach from critical discourse analysis. Hampshire: Macmillan. https://doi.org/10.1007/978-0-230-20968-8 
Riechmann, J. (1995). Desarrollo sostenible: La lucha por la interpretación. En J. Riechman, J. M. Naredo, R. B. Gómez, A. Esteban, C. Taibo, J. C. Rodríguez y J. Nieto (Eds.), De la economía a la ecología (pp. 11-36). Madrid: Trotta.

Solber, J. (2013). Contribución de las cuestiones sociocientíficas al desarrollo del pensamiento crítico II. Revista Eureka sobre Enseñanza y Divulgación de las Ciencias, 1O(2), 171-181. https://doi.org/10.25267/Rev_Eureka_ensen_divulg_cienc.2013.v10.i2.03

Tan, S., Kindelan, E. M. y O’Halloran, K. L. (2012). Multimodal analysis image. Singapur: Multimodal Analysis Company.

Toledo Jofré, M., Magendzo Kolstrein, A., Gutiérrez Gianella, V. e Iglesias Segura, R. (2015). Enseñanza de "temas controversiales" en la asignatura de historia y ciencias sociales desde la perspectiva de los profesores. Estudios Pedagógicos, 41(1), 275-292. https://doi.org/10.4067/S0718-07052015000100016

Touriñán López, J. M. (2008). La educación en valores como uso y construcción de experiencia axiológica. En J. M. Touriñán López (Dir.), Educacion en valores, educación intercultural y formación para la convivencia pacífica (pp. 2-11). La Coruña: Netbiblo.

UNESCO. (2014a). Declaración de Aichi-Nagoya sobre la educación para el desarrollo sostenible. AichiNagoya. París: UNESCO.

UNESCO. (2014b). Página web principal de la Organización de las Naciones Unidas para la Educación, la Ciencia y la Cultura. Recuperado de http://www.unesco.org/new/es/ourpriorities/sustainable-development/

UNESCO. (2015). Educación para la ciudadanía mundial. Temas y objetivos de aprendizaje. Nueva York, NY: UNESCO.

Vilches, A. y Gil Pérez, D. (2015). Ciencia de la sostenibilidad: ¿Una nueva disciplina o un nuevo enfoque para todas las disciplinas? Revista Iberoamericana de Educación, 69(1), 39-60.

Vilches, A. y Gil Pérez, D. (2016). La transición a la sostenibilidad como objetivo urgente para la superación de la crisis sistémica actual. Revista Eureka sobre Enseñanza y Divulgación de las Ciencias, 13(2), 395-407.

https://doi.org/10.25267/Rev_Eureka_ensen_divulg_cienc.2016.v13.i2.12 


\section{Apéndice 1. Encuesta relacionada con la EDS}

1. ¿Te parece adecuado introducir temas relacionados con la justicia ambiental y la sostenibilidad en la enseñanza universitaria con independencia de la carrera que se estudie?

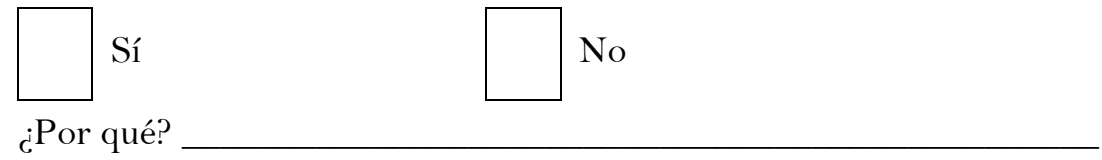

2. ¿Conocías los Objetivos del Desarrollo Sostenible antes de que se mencionaran en la asignatura Lengua Inglesa V?

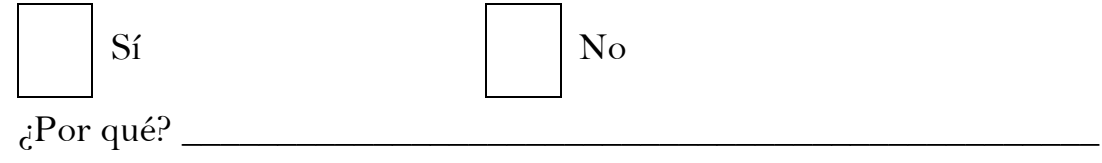

3. ¿Te consideras una persona ecologista y preocupada por el medio ambiente?

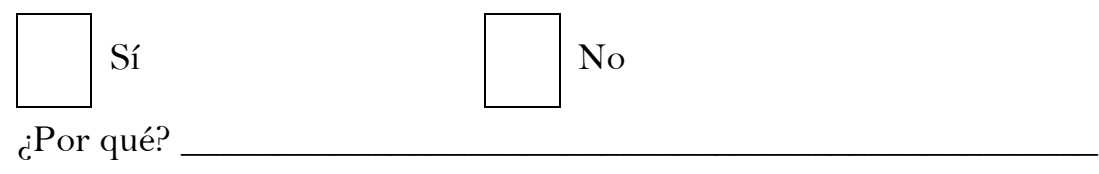

4. ¿Has aprendido vocabulario y aspectos relacionados con la ecología y la sostenibilidad al realizar las actividades propuestas?

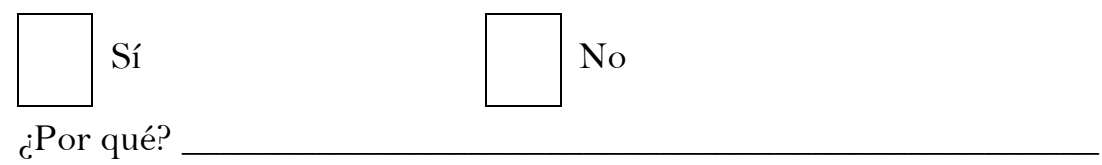

5. ¿Cuáles de las siguientes competencias sociales consideras que has adquirido más al realizar las actividades enmarcadas en la EDS?
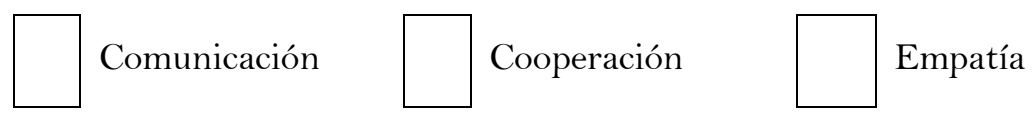

\section{Breve CV de la autora}

\section{María Martínez Lirola}

Doctora en Filología Inglesa y Profesora Titular de Filología Inglesa de la Universidad de Alicante. Además, es Research Fellow del Departamento de Lingüística y Lenguas Modernas de la Universidad de Sudáfrica (UNISA). Ha dirigido varios proyectos de investigación sobre Análisis Crítico del Discurso e innovación educativa financiados por entidades públicas. Sus principales líneas de investigación son el Análisis Crítico del Discurso, la Gramática Sistémica Funcional y la Lingüística Aplicada. Ha publicado alrededor de un centenar de publicaciones que incluyen libros, artículos en revistas nacionales e internacionales, capítulos de libro, ponencias en congresos, reseñas y trabajos de divulgación, además de haber creado y dirigir el grupo de investigación "Análisis Crítico del Discurso Multimodal". ORCID ID: 0000-0002-6427-425X. Email: maria.lirola@ua.es 